\title{
Modellierung des elektrischen Feldes für Anordnungen mit Singularitäten
}

\author{
D. Mente, M. Greiff, and W. Mathis \\ Institut für Theoretische Elektrotechnik, Universität Hannover, Applestraße 9A, 30167 Hannover, Deutschland
}

\begin{abstract}
Zusammenfassung. Mit Hilfe von weitverbreiteten numerischen Methoden wie BEM/FEM lassen sich Singularitäten im Feldverlauf nur bedingt berücksichtigen. Es sollen daher zwei alternative numerische Feldberechnungsmethoden vorgestellt werden, die sich insbesondere für Spitzengeometrien eigenen, wie sie bei Rasterkraftmikroskopen eingesetzt werden. Dabei soll ein weiteres Augenmerk auf die Eignung der Kopplung der vorgeschlagenen Methoden an andere Feldberechnungsmethoden gerichtet werden.
\end{abstract}

Abstract. Modern numerical methods for field calculations are having problems dealing with singularities correctly. This paper provides two alternative methods that are able to handle electric fields including singularities in tip-like configurations. These configurations occur in atomic force microscopes. Another focus will be the suitability of coupling the presented methods with usual numerical methods.

\section{Einleitung}

In der Forschung und Entwicklung spielt die numerische Berechnung von Feldern eine große Rolle. Moderne Feldberechungsmethoden können jedoch nur bedingt Singularitäten in der Feldverteilung berücksichtigen. Doch gerade diese Singularitäten sind in einigen Anordnungen von großer Bedeutung. Hier sollen Ansätze am Beispiel einer Simulation eines elektrostatischen Kraftmikroskopes (Bhushan, 2003) vorgestellt werden. Dieses, der Gruppe der Mikro ElektroMechanischen Systeme (MEMS) zuzuordnende Mikroskop, kann elektrostatische Felder messen. Neben Singularitäten enthält das Modell einerseits extrem feine Strukturen im Nanometerbereich und andererseits Strukturen im Mikrometeroder Millimeterbereich. Diese Multi-Skalen-Eigenschaft erfordert besondere Beachtung in der numerischen Modellie-

Correspondence to: D. Mente

(dmen@tet.uni-hannover.de) rung. Es werden zwei Methoden vorgestellt, die diese Anordnungen mit Singularitäten modellieren. Des Weiteren soll die Eignung der Methoden zur Kopplung an andere numerische Berechnungsmethoden, z.B. der Finite-ElementeMethode (FEM), beispielsweise in Greiff (2004), herausgestellt werden. Die betrachteten Probleme sind zweidimensional, doch lassen sich die Methoden einfach auf dreidimensionale Probleme erweitern.

\section{Elektrostatisches Kraftmikroskop}

In diesem Beitrag soll das elektrische Feld eines elektrostatischen Kraftmikroskopes (EFM) mittels zweier verschiedener Methoden berechnet werden. Zur Verdeutlichung soll zunächst kurz die Funktionsweise dieser Mikroskope erklärt werden. Sie erlauben die Abtastung von Oberflächen bis in den Nanometerbereich hinein. Dazu wird eine an einem biegsamen Ausleger (engl. "Cantilever") befestigte Nadel (engl. "Tip" oder "Probe") zeilenweise über ein Prüfstück gefahren. Dieses Prüfstück wird auch als Probe (engl. "Sample") bezeichnet. Dabei wirken verschiedene Kräfte auf den Ausleger. Im Falle des EFM sind dies insbesondere elektrische Kräfte, die durch Potentialunterschiede zwischen Ausleger und Prüfstück entstehen. Durch die ansetzenden Kräfte wird der Ausleger verbogen. Diese Auslenkung wird mittels optischer Messverfahren gemessen. Dazu wird ein Laser eingesetzt, der an der Spitze des Auslegers abhängig von der Auslenkung reflektiert wird. Eine 4-Segment Photodiode dient der Erfassung dieser Reflektion. Mikroaktuatoren regeln die Position des Auslegers anschließend so nach, dass sich der Ausleger wieder in seiner ursprünglichen Lage befindet. Aus der nötigen Nachregelung der Position mittels der Aktutatoren wird auf die wirkende Kraft geschlossen.

Beim EFM ist das elektrische Feld im Bereich der Spitze und damit im Bereich der Singularität am größten, womit auch die elektrische Kraft dort am größten ist. Der Exaktheit 


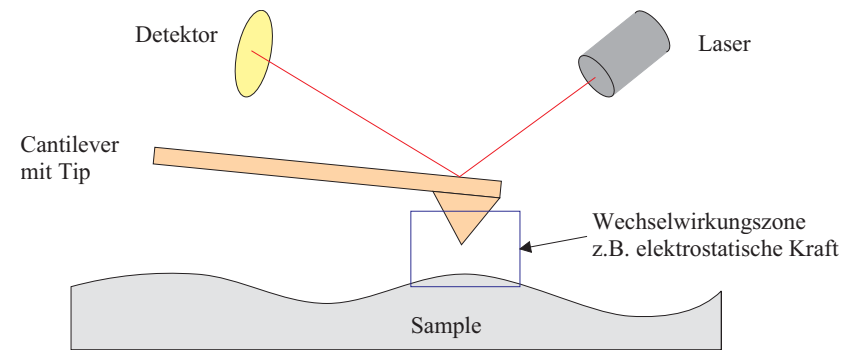

Abb. 1. Aufbau des Electrostatic Force Microscope.

des Feldes bei der Modellierung kommt in diesem Bereich also große Bedeutung zu. Daraus folgend muss die Singularität bei der Modellierung des elektrischen Feldes miteinbezogen werden. Zur Simulation des EFM muss diese Kraft aus dem Feld berechnet werden. Die Feldverteilung kann beispielsweise aus den beiden nun folgenden numerischen Methoden gewonnen werden.

\section{Das Verhalten des Feldes im Bereich von Kanten}

Für das Verständnis der Problematik der in dieser Arbeit benutzten Anordnung ist es wichtig zu verstehen, wie sich das Feld speziell in der Nähe von Ecken und Kanten verhält. Eine Ecke oder Kante kann mittels zwei sich treffender Halbebenenen modelliert werden. Die hergeleiteten Methoden werden in dieser Arbeit auf zweidimensionale Probleme angewandt. Eine prinzipielle Anordnung mit den hier benutzten Bezeichnungen ist in Abb. 2 dargestellt.

Im betrachteten Gebiet gilt $\rho=0$, d.h. das Gebiet ist ladungsfrei. Daher ist die Laplace-Gleichung zu lösen, um die Potentialverteilung zu erhalten. Werden zweckmäßigerweise Polarkoordinaten gewählt, sieht nach Jackson (2002) die Laplace-Gleichung folgendermaßen aus:

$\frac{1}{\rho} \frac{\partial}{\partial \rho}\left(\rho \frac{\partial \varphi}{\partial \rho}\right)+\frac{1}{\rho^{2}} \frac{\partial^{2} \varphi}{\partial \phi^{2}}=0$.

Zunächst erfolgt ein Produktansatz nach Bernoulli:

$\varphi(\rho, \phi)=R(\rho) \cdot \Phi(\phi)$,

mit $R(\rho)$ und $\Phi(\phi)$ als gesuchte Funktionen von nur einer Veränderlichen. Nach Separation der Variablen, der Lösung der enstehenden Differentialgleichungen sowie der Anpassung der Konstanten ergibt sich für Potential und Feld:

$$
\begin{aligned}
& \varphi(\rho, \phi)=V+\sum_{n=1}^{\infty} c_{n} \rho^{\frac{n \pi}{\beta}} \sin \left(\frac{n \pi \phi}{\beta}\right) \\
& E_{\rho}(\rho, \phi)=-\frac{\partial \varphi}{\partial \rho}=-\sum_{n=1}^{\infty} c_{n} \frac{n \pi}{\beta} \rho^{\frac{n \pi}{\beta}-1} \sin \left(\frac{n \pi}{\beta} \phi\right)
\end{aligned}
$$

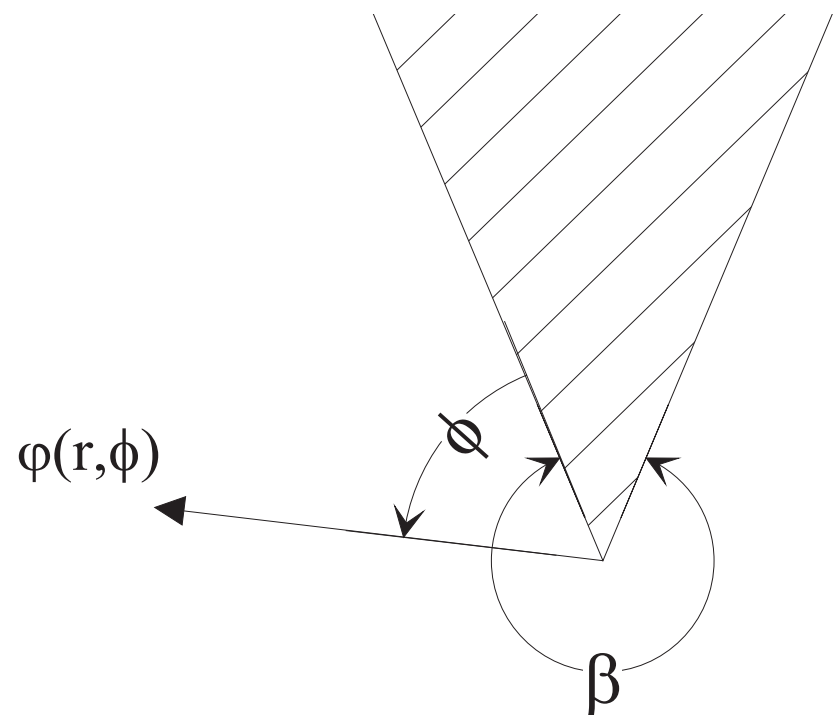

Abb. 2. Schnitt zweier Ebenen, die eine Ecke bilden.

und

$E_{\phi}(\rho, \phi)=-\frac{1}{\rho} \frac{\partial \varphi}{\partial \phi}=-\sum_{n=1}^{\infty} c_{n} \frac{n \pi}{\beta} \rho^{\frac{n \pi}{\beta}-1} \cos \left(\frac{n \pi}{\beta} \phi\right)$,

mit dem Potential $V$ auf der Spitze. Hier ist zu erkennen, dass das Feld für Winkel $\beta(>) \pi$ singulär wird, denn der Term $\rho^{\frac{n \pi}{\beta}-1}$ ist dann für $n=1$ und $r \rightarrow \infty$ unendlich. Dieser Term modelliert also die an der Spitze entstehende Singularität.

\section{Methode der fundamentalen Lösungen}

Die Methode der fundamentalen Lösungen (MdfL, siehe Li, 1990) greift die Formeln (3), (4) und (5) auf. Diese Gleichungen enthalten unendliche Summen und sind daher nicht exakt in einem maschinellen Programm zu implementieren. Da die Koeffizienten bei realen Potential- und Feldverteilungen jedoch rasch gegen null konvergieren, besteht die Möglichkeit die unendlichen Reihen abzuschneiden. Dadurch entstehen endliche Reihen, mit denen sich das Feld näherungsweise bestimmen lässt. Diese Lösung basiert auf dem Separationsansatz in 2D mit den dort getroffenen Randbedingungen. Daraus ergibt sich auch, dass die Ebenen, mit denen die Messspitze modelliert wurde, unendlich ausgedehnte Halbebenen sein müssen. Die Messspitze ist bei dieser Modellierung unendlich ausgedehnt. Ein Fehler bei der Feldbestimmung des Mikroskopes ist daher in Kauf zu nehmen. Deshalb folgt, dass diese Methode nur für Felder in der Nähe von Ecken und Kanten geeignet ist. Denn nur wenn der Bereich in dem eine Lösung der Laplace-Gleichung gesucht wird klein gegenüber der Gesamtausdehnung der Spitze ist, wird der Fehler vernachlässigbar. 


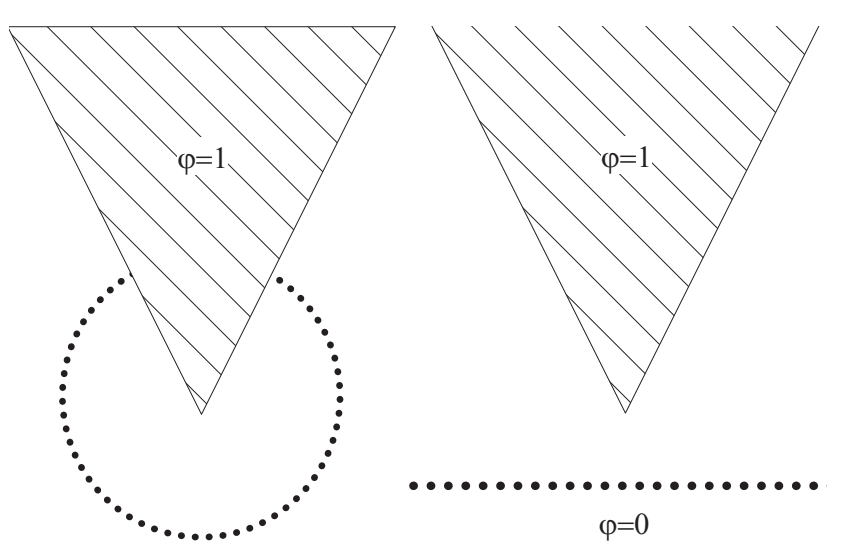

Abb. 3. Verwendete Ersatzanordnungen.

Die Koeffizienten lassen sich über Randbedingungen bestimmen. Diese bestehen aus Potentialvorgaben oder Komponenten des Feldes in diskreten Punkten. Das Einsetzen der einzelnen Randbedingungen in (3), (4) und (5) führt zu einem Gleichungssystem $\mathbf{A x}=\mathbf{b}$. Dabei stellt $\mathbf{x}$ einen Vektor dar, der die gesuchten Koeffizienten $c_{n}$ enthält. Der Position der Randbedingungen kommt hier eine große Bedeutung zu. Sind die Bedingungen über einen kleinen Winkelbereich verteilt, so kann die Vorgabe, Randbedinungen exakt zu erfüllen, zu einem "Aufschwingen" der Koeffizienten führen. Die Lösung wird dann unbrauchbar. Hilfreich ist in diesen Fällen die Überbestimmung des Gleichungssystems. Dann wird zur Lösung die Methode des minimalen quadratischen Fehlers angewandt. Dies kann jedoch durch gleichmäßige Verteilung der Randbedingungen über den zur Verfügung stehenden Winkelbereich vermieden werden. Sinnvollerweise wird diese Art der Verteilung benutzt, wenn die Methode der fundamentalen Lösungen mit anderen numerischen Methoden gekoppelt werden soll (Abb. 3, links). Eine weitere Verfälschung der Lösung durch Abschneiden der unendlichen Reihe entsteht, wenn eine Ersatzanordnung nach Abb. 3, rechts verwendet wird. Über einen Winkelbereich sind konstante Randbedingungen, hier $\varphi=0$ vorgegeben. Es kommt Aufgrund der endlichen Anzahl der verfügbaren Moden zu Überschwingen, wie sie ähnlich bei Fouriertransformationen von Rechtecksignalen entstehen, wenn die Anzahl der Terme der Reihe begrenzt werden. Die dadurch hervorgerufenen Störungen sind jedoch sehr gering. Bei 100 Randbedingungen entsprechend Abb. 3, rechts angeordnet ergibt sich ein maximaler Fehler des Potentials in der Größenordnung von $\sim 10^{-10}$.

\section{Ersatzladungsmethode}

Das der Ersatzladungsmethode ("Charge Simulation Method”, CSM) zugrunde liegende Prinzip ist sehr einfach. Ist eine Anzahl diskreter Ladungen beliebigen Typs in einem
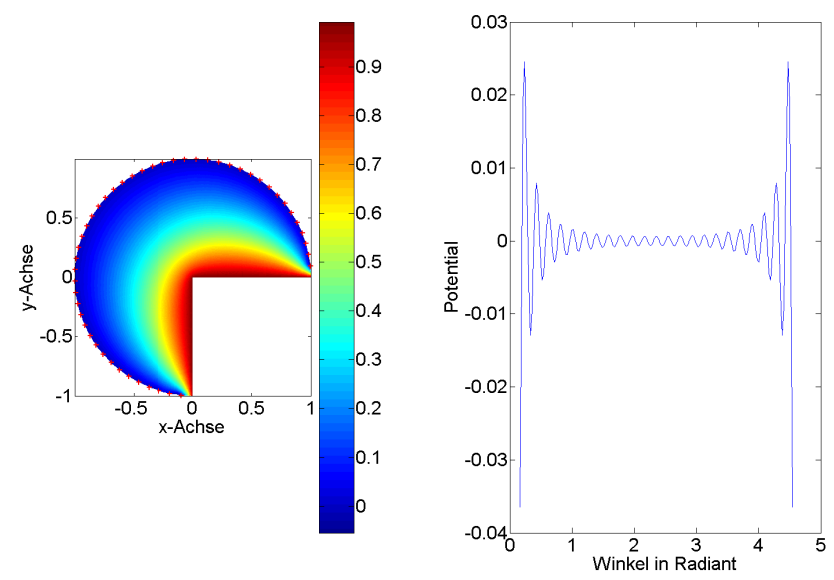

Abb. 4. MdfL: Kreisförmige Anordnung der Randbedingungen $\varphi=$ 0 und Verlauf des Potentials zwischen den Randbedingungen.

Raum bekannt, so lässt sich das Potential in einem Punkt $c_{i}$ durch Summation der aus den Ladungen resultierenden Einzelpotentiale in diesem Punkt berechnen (Überlagerungsprinzip). So können Randbedingungen durch fiktive Ladungen ersetzt werden. Aufgrund der Verwandtschaft der Ersatzladungsmethode zur analytischen Spiegelungsmethode werden diese fiktiven Ladungen auch Spiegelungsladungen ("image charges") genannt. Bei der Beschränkung auf zweidimensionale Probleme bleiben als mögliche Ladungstypen nur noch unendliche Linienladungen erhalten. Der Wert der Linienladung $\lambda$ muss den gegebenen Randbedingungen entsprechend gewählt werden. Bei der Ersatzladungsmethode sind die Randbedingungen bestimmte Werte, die eine Funktion an bestimmten Punkten, sogenannten Konturpunkten annimmt. Die Konturpunkte bilden beispielsweise die Konturen der Leiter nach. Das Potential $\varphi_{i}$ ergibt sich nach

$\varphi_{i}=\sum_{j=1}^{n} P_{i j} \lambda_{j}$

$P_{i j}$ ist ein vom verwendeten Ladungstyp abhängiger Term, der in diesem Zusammenhang auch Potentialkoeffizient genannt wird. Beim hier benutzen Typ unendliche Linienladung entspricht er

$P_{i j}=\frac{1}{2 \pi \varepsilon_{0}} \ln \frac{r_{i j}}{r_{0}}$,

wobei $r_{i j}$ der Entfernung von der Linienladung $\lambda_{j}$ zum Konturpunkt $c_{i}$ entspricht. Sind beispielsweise drei Ladungen in einem Raum platziert, lässt sich das Potential $\varphi_{i}$ in einem Punkt $c_{i}$ wie folgt ausdrücken:

$\varphi_{i}=\frac{\lambda_{1}}{2 \pi \varepsilon_{0}} \ln \frac{r_{i 1}}{r_{0}}+\frac{\lambda_{2}}{2 \pi \varepsilon_{0}} \ln \frac{r_{i 2}}{r_{0}}+\frac{\lambda_{3}}{2 \pi \varepsilon_{0}} \ln \frac{r_{i 3}}{r_{0}}$.

Sind die drei Ladungen $\lambda_{1,2,3}$ als unbekannt vorausgesetzt, so werden zwingend drei Konturpunkte $c_{1,2,3}$ mit bekannten Potentialen $\varphi_{1,2,3}$ zur exakten Lösung der Gleichung 


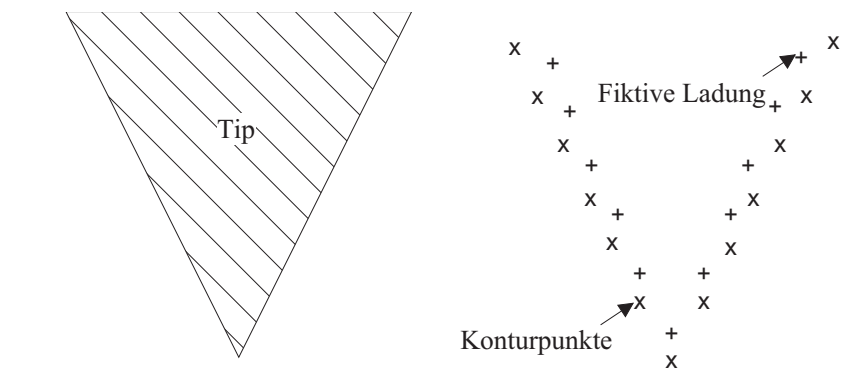

Sample

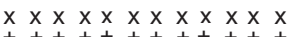

Abb. 5. Spitze des EFM und modellhafte Anordnung für Ersatzladungsmethode.

benötigt. Damit ist es möglich, die Ladung und das Potential an jeder Randbedingung quantitativ miteinander in Beziehung zu setzen. Gleiches ist mit den Komponenten des Feldes möglich. Bei der Ersatzladungsmethode werden die fiktiven Landungen außerhalb des Raumes platziert, in dem eine Lösung der Laplacegleichung gesucht wird. Wenn ein Konturpunkt sich auf einer Oberfläche eines Leiters befindet, dann soll das Potential dort gleich dem Potential des Leiters sein. Wendet man dieses Prinzip auf alle m Konturpunkte und $\mathrm{n}$ Ladungen an, so ergibt sich folgendes Gleichungssystem:

$\left[\begin{array}{ccc}P_{11} & \cdots & P_{1 n} \\ \vdots & \ddots & \vdots \\ P_{m 1} & \cdots & P_{m n}\end{array}\right]\left(\begin{array}{c}\lambda_{1} \\ \vdots \\ \lambda_{n}\end{array}\right)=\left(\begin{array}{c}\varphi_{1} \\ \vdots \\ \varphi_{m}\end{array}\right)$

Angenommen, man hat die in Abb. 5 gegebene Anordnung einer Spitze des Rasterkraftmikroskopes, dann werden zur Feldberechnung mittels der Ersatzladungsmethode die vorhanden Ladungen auf der Oberfläche der Leiter durch n fiktive Ladungen ersetzt. Um den Wert der Ladungen zu bestimmen, werden nun m Konturpunkte auf der Oberfläche der Leiter platziert. Hier müssen die überlagerten Potentiale aller fiktiven Ladungen einen bestimmten Wert, in diesem Fall das Potential des Leiters, haben. Im Bereich auf dem Leiter zwischen den Konturpunkten gilt dies nicht. Daher ist durch Einsetzen in Gl. (6) zu überprüfen, ob das gewünschte Genauigkeitskriterium entlang des Leiters erfüllt ist.

Eine größere Anzahl an Ladungen und Konturpunkten erhöht die Genauigkeit der Simulation. Auch eine größere Anzahl an Konturpunkten als Ladungen kann die Genauigkeit erhöhen (Überbestimmung). Hierbei wird die Methode des geringsten Fehlerquadrates eingesetzt, wobei eine zusätzliche Gewichtung nach Steinbigler (1968) mit einbezogen werden kann, um in einem kritischen Bereich eine genauere Lösung zu erhalten. Der Platzierung der fiktiven Ladungen und Konturpunkte kommt bei der Ersatzladungsmethode eine wesentliche Bedeutung zu. Die Lage der Punkte bestimmt die Genauigkeit der späteren Ergebnisse der Simu-

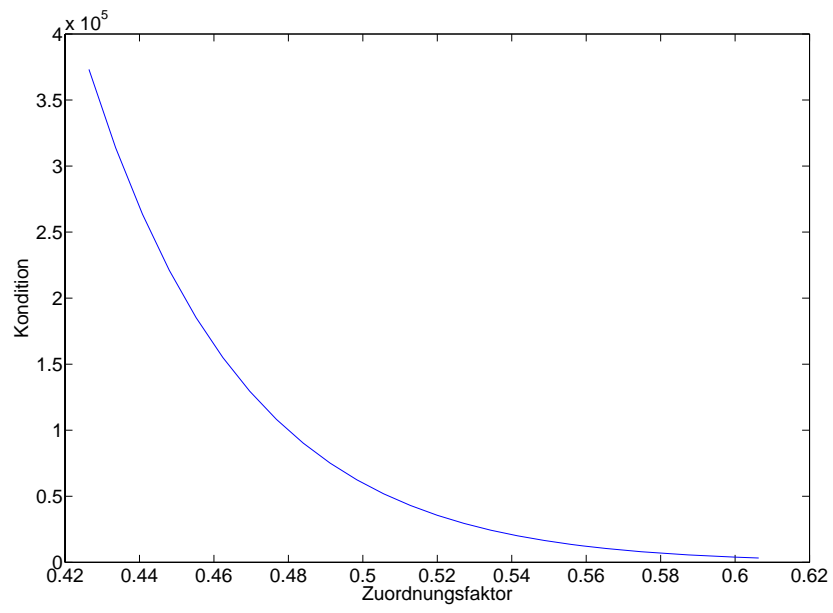

Abb. 6. Verbesserung der Kondition bei Vergrößerung von $f_{a}$.

lation. Die Konturpunkte bilden die Konturen der Leiter nach und sind deshalb dort zu setzen. Ausnahme sind Räume mit mehreren Dielektrika, bei denen zusätzlich Ladungen an den Flächen zu platzieren sind, an denen verschiedene Dielektrika aufeinander treffen.

Allerdings steigt der Fehler der Simulation, wenn sich die Ladung zu nah an der Randfläche befindet, weil dann die Krümmung der Äquipotentialflächen der Linienladung in den Vordergrund tritt. In diesem Fall wird dann der Potentialunterschied auf der ehemaligen Leiteroberfläche und zwischen den Konturpunkten groß. Ist die Ladung weiter von der Oberfläche entfernt, ist diese Krümmung weniger ausgeprägt. Bei der Auswertung der numerischen Simulationsergebnisse tritt ein zweiter zu berücksichtigender Faktor auf: Je größer der Abstand der Ladung von den Konturpunkten desto schlechter wird die Kondition des Problems. In der numerischen Mathematik beschreibt man mit der Kondition die Abhängigkeit der Lösung eines Problems von der Störung der Eingangsdaten. Die Konditionszahl stellt ein Maß für diese Abhängigkeit dar. Sie beschreibt den Faktor, um den Eingangsfehler im ungünstigsten Fall verstärkt werden.

Die Kondition der Matrix $\mathbf{P}$ (Gl. 9) hängt von zwei Faktoren ab: Der Entfernung $r_{i j}$ der Ladungen zu den Konturpunkten und der Entfernung $r_{0}$ der Ladung zum Nullpunkt. Sie sind in den Potentialkoeffizienten (Gl. 7) enthalten. Ist der Nullpunkt weit außerhalb des betrachteten Gebietes $r_{0}>>r_{i j}$, so ist der Term $r_{i j} / r_{0}$ sehr klein. Die resultierenden Potentialkoeffizienten nähern sich dann mit steigender Entfernung des Nullpunktes aneinander an, wobei sich die Kondition verschlechtert. Der Nullpunkt ist also in die Nähe der Anordnung zu legen. Der gleiche Effekt ist bei der Entfernung der Ladungen zu den Konturpunkten $r_{i j}$ zu beobachten. Dabei spielt der Abstand einer Ladung zu dem korrespondierenden Konturpunkt im Verhältnis zu dem Abstand zu allen anderen Konturpunkten eine Rolle. Ist der Abstand 
einer Ladung zum korrespondierenden Konturpunkt groß, so ist er auch zu allen anderen Konturpunkten verhältnismäßig groß. Dann ähneln sich auch die Potentialkoeffizienten sehr und die Kondition der Matrix $\mathbf{P}$ wird schlecht. Ist der Abstand zum korrespondierenden Konturpunkt sehr gering, ist im Verhältnis dazu der Abstand zu den anderen Konturpunkten $r_{i j}$ groß. Die resultierenden Koeffizienten unterscheiden sich dann immer stärker und die Kondition verbessert sich. Um ein gut konditioniertes Problem zu erreichen sind die Ladungen also möglichst nah an den Konturpunkten zu platzieren.

Es sind einerseits die Ladungen nah an den Konturpunkten zu platzieren, um die Kondition zu verbessern. Andererseits sollten die Ladungen in möglichst großer Entfernung zu den Konturpunkten sein, damit die Potentialunterschiede auf den Leitern, bedingt durch die gekrümmten Äquipotentialflächen der Linienladungen, nicht zu groß werden. Diese gegenläufigen Aspekte gilt es zu minimieren. Dazu wird zunächst der Zuordnungsfaktor $f_{a}$ aus Singer (1974) definiert. Er beschreibt das Verhältnis der Entfernung der Ladungen untereinander zu der Entfernung der Ladungen zu den Konturpunkten. In Malik (1989) wird zusammenfassend ein Faktor von $0,7 \leq f_{a} \leq 2$ genannt. Doch beeinflusst die simulierte Geometrie diesen Faktor. Daher ist $f_{a}$ für jede Anordnung unterschiedlich. Zur Bestimmung von $f_{a}$ für die verwendete Geometrie wurden verschiedene Simulationen ausgeführt. Zum einen wurde die Kondition für verschiedene Werte des Zuordnungsfaktors aufgezeichnet (Abb. 6). Dem gegenübergestellt ist die Standardabweichung des Potentials entlang des Leiters der das Prüfstück (Abb. 7). Aus den Ergebnissen dieser Simulation ist ersichtlich, das ein Optimum um $f_{a} \approx 0,6$ besteht.

Abbildung 8 zeigt ein Ergebnis einer Simulation des Cantilevers. Es ist der Betrag des Feldes dargestellt. Benutzt wurden 72 Ladungen und 72 Konturpunkte. Wie zu erwarten führt die Nachbildung des Cantilever führt an den Kanten zu mehreren Überhöhungen an den Kanten des Auslegers. Diese sind jedoch aufgrund der geringeren Potentialunterschiede weniger stark ausgeprägt.

\section{Diskussion}

Es wurden zwei numerische Verfahren zur zweidimensionalen Feldberechnung des elektrostatischen Kraftmikroskopes oder Teilen davon vorgestellt. Andere numerische Methoden (FEM/BEM) erfordern im Bereich von Singularitäten eine feine Diskretisierung des Raumes/Oberfläche. Die hier vorgestellten Methoden erlauben eine Modellierung des Feldes mit weniger Aufwand und führen zu schnelleren Lösungen.

Eine der Methoden ist die Ersatzladungsmethode. Ausgangspunkt bildeten hier die Arbeiten von Steinbigler (1968) und Singer (1974). Prinzipiell basiert die Ersatzladungsmethode auf dem Ersetzen von Randbedingungen durch Ladungen. Durch Lösen eines linearen Gleichungssystems können

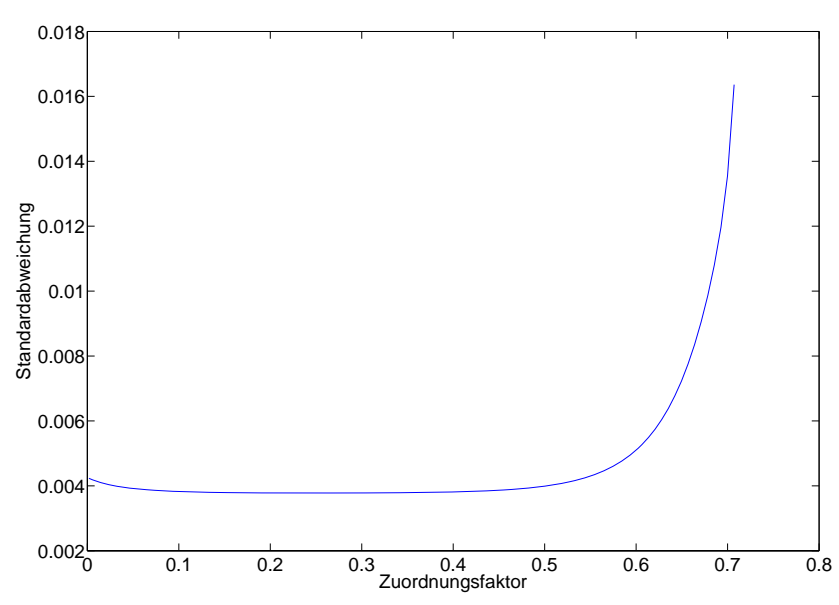

Abb. 7. Verschlechterung der Standardabweichung bei Vergrößerung von $f_{a}$.

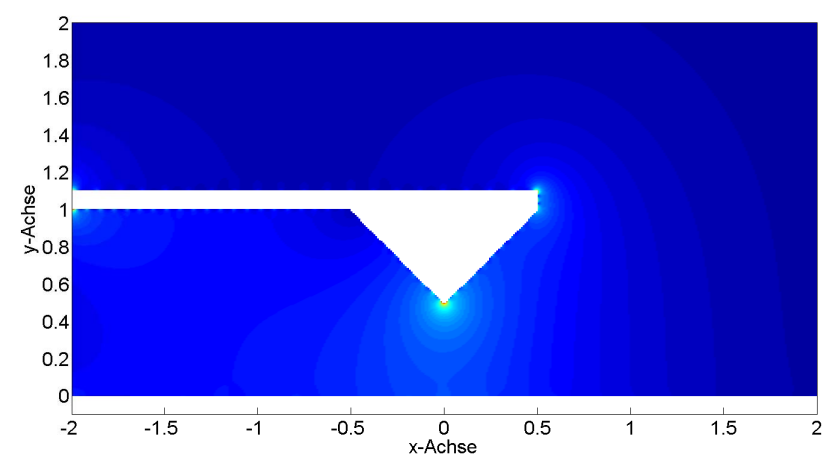

Abb. 8. Betrag des elektrischen Feldes nach Simulation des Cantilevers mit Probe.

die Ladungen so bestimmt werden, dass sie die Randbedingungen erfüllen. Hierbei kommt der Position der Ladungen und Konturpunkte entscheidende Bedeutung $\mathrm{zu}$, denn von ihnen hängt maßgeblich die Güte der Lösung ab. Ebenfalls untersucht wurde die Kondition des Gleichungssystems in Abhängigkeit zur Position der Ladungen und Konturpunkte sowie das Verhalten bei Überbestimmung des Gleichungssystems. Insgesamt scheint die Ersatzladungsmethode zur Kopplung an andere numerische Methoden (z.B. FEM) geeignet zu sein. Bei überbestimmten Gleichungssystemen lieBe sich die Güte der Simulation durch Einführung von Gewichtungsfaktoren verbessern, die den Fehler in bestimmten Bereichen minimieren. Ein solcher Bereich kann z.B. die Singularität sein.

Die zweite implementierte Methode ist die Methode der fundamentalen Lösungen. Sie basiert auf den Reihen (3), (4) und (5) die sich aus dem Produktansatz nach Bernoulli ergeben. Dabei wird die Singularität direkt in die Ansatzfunktionen miteinbezogen. Aufgrund der verwendeten Ansatzfunk- 
tionen kann es bei ungünstiger Wahl der Randbedingungen zu Überschwingen des Potentials kommen. Genaue Analysen der MdfL ergaben, dass sie eine effiziente Methode zur Berechnung des Feldes ist. Auch scheint diese Methode für hybride Berechnungsmethoden besonders geeignet, da dort die Randbedingungen günstig verteilt sind und die Kondition gut ist. Ein Teil der Geometrie ist bereits in der Ausgangsfunktion enthalten, weshalb die MdfL auf bestimmte Anordnungen beschränkt ist. Daraus ergibt sich auch, dass sie sich nur auf Probleme in der Nähe von Kanten und Ecken anwenden lässt. Beiden hier implementierten Methoden ist gemein, dass die Position der Randbedingungen einem großen Einfluss auf die Genauigkeit der Simulation zukommt. Die Abweichungen von den Randbedingungen sind bei vergleichbarer Gleichungsgröße und Anordnung bei der MdfL geringer als bei der Ersatzladungsmethode.

\section{Literatur}

Bhushan, B.: Handbook of Nanotechnology, Springer-Verlag, Berlin, Heidelberg, New York, 2003.

Greiff, M., Bala, U. B., and Mathis, W.: A Staggered ALE Approach for Coupled Electromechanical Systems, SCEE Capo D'Orlando, 5.-9. September 2004.

Jackson, J. D.: Klassische Elektrodynamik, Walter De Gruyter Berlin, New York, 3. Auflage, 2002.

Malik, N. H.: A rieview of the charge simulation method and its applications, IEEE Transactions on Electrical Insulations, 24, 1, 3-20, 1989.

Singer, H., Steinbigler, H., and Weiss, P: A charge simulation method for the calculation of high voltage fields, IEEE Transactions on Power Apparatus and Systems, 88, 1802-1814, 1974.

Steinbigler, H.: Anfangsfeldstärken und Ausnutzungsfaktoren rotationssymetrischer Elektrodenanordnungen, Dissertation, TU München, 1968.

$\mathrm{Zi} \mathrm{Cai} \mathrm{Li:} \mathrm{Numerical} \mathrm{methods} \mathrm{for} \mathrm{elliptic} \mathrm{problems} \mathrm{with} \mathrm{singulari-}$ ties, World Scientific, Singapure, 1990. 